\title{
CARACTERÍSTICAS DE LA CULTURA ORGANIZACIONAL EN LA FACULTAD DE ADMINISTRACIÓN DE EMPRESAS DE LA UNIVERSIDAD NACIONAL DEL CENTRO DEL PERÚ
}

\author{
CHARACTERISTICS OF ORGANIZATIONAL CULTURE IN THE BUSINESS ADMINISTRATION \\ FACULTY UNIVERSIDAD NACIONAL DEL CENTRO DEL PERU
}

\author{
Isac Fernando Espinoza Montes \\ Facultad de Administración de Empresas de la Universidad Nacional del Centro del Perú.
}

\section{RESUMEN}

Partiendo de la interrogante; ¿cuáles son las características de la cultura organizacional en la Facultad de Administración de Empresas de la Universidad Nacional del Centro del Perú?, el estudio pretendió determinar y describir las características de la cultura organizacional que existe en la Facultad de Administración de Empresas de la UNCP para proponer un algoritmo para el cambio cultural. Utilizando los métodos; deductivo y el analítico sintético, las técnicas de la encuesta y la estadística descriptiva (media) y el estadístico t, con un nivel de significancia de 0.05 y grado de libertad de 29 , el estudio concluye que; se rechaza la hipótesis nula y se acepta la hipótesis de investigación, evidenciando que "La cultura organizacional de la Facultad de Administración de Empresas de la Universidad Nacional del Centro del Perú, se singulariza por ser débil; caracterizado por la deficiente promoción de valores y clima de confianza, poca identidad, deficiente flujo de la información, clima organizacional desfavorable, carencia de motivación". A partir del análisis, el estudio presenta un algoritmo para el cambio cultural en la Facultad a través de las fases como: diagnosticar la cultura actual, definir los cambios culturales, identificar los comportamientos deseados, desarrollar programas para lograr compromisos, modelar roles de los líderes, alinear sistemas y procesos.

Palabras clave: cultura organizacional, algoritmo, cambio cultural

\begin{abstract}
Based on the question, What are the characteristics of the organizational culture in the Faculty of Business Administration of the Universidad Nacional del Centro?, The study sought to identify and describe the characteristics of the organizational culture that exists in the Management Business Faculty of UNCP to propose an algorithm for cultural change. Using the methods, deductive and analytical synthetic survey techniques and descriptive statistics (mean) and the t statistic, with a significance level of 0.05 and freedom degree of 29, the study concludes that; hypothesis null is rejected and research hypothesis is accepted, showing that "the organizational culture of the Faculty of Business Administration of the Universidad Nacional del Centro del Perú is singled out for being weak, characterized by poor promotion and confidence values, little identity, poor information flow, unfavorable organizational climate, lack of motivation. From the analysis, the paper presents an algorithm for cultural change in the Faculty through phases like: Diagnose the current culture, defining cultural changes, identify desired behaviors, develop programs to achieve commitments, roles of leaders modeling, aligning systems and processes.
\end{abstract}

Keywords: organizational culture, algorithm, cultural change

\section{INTRODUCCIÓN}

La interrogante de investigación que expresa el problema, ¿cuáles son las características de la cultura organizacional en la Facultad de 
Administración de Empresas de la Universidad Nacional del Centro del Perú?, es de carácter descriptivo ex post facto y se sustenta en el planteamiento de Gibson et al. (2006),donde sostiene que cultura organizacional "es lo que los empleados perciben y la manera en que esta percepción crea un patrón de creencias, valores y expectativas".

La definición precedente es profundizada por Davis (1993), quien plantea que las organizaciones, al igual que las huellas digitales, son siempre singulares y puntualiza que poseen su propia historia, comportamiento, proceso de comunicación, relaciones interpersonales, sistema de recompensa, toma de decisiones, filosofía y mitos que, en su totalidad, constituyen la cultura, que agrupadas y medidas en dimensiones (ver figura 1), puede singularizar las culturas como débiles o fuertes.

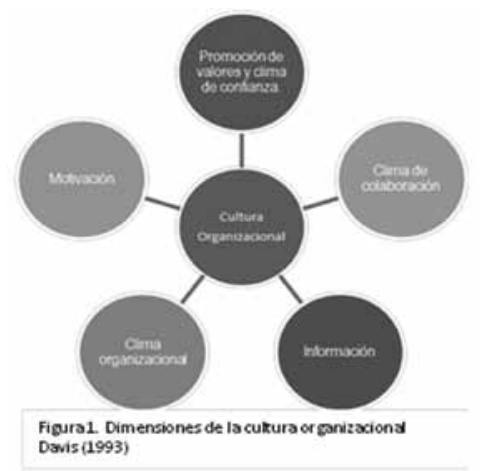

Basado en la fundamentaciónteórica y empírica que antecede, la investigación postulò que; la cultura organizacional de la Facultad de Administración de Empresas de la U.N.C.P., se singulariza por ser débil; caracterizado por la deficiente promoción de valores y clima de confianza, poca Identidad, deficiente flujo de la información, clima organizacional desfavorable, carencia de motivación y se propuso; determinar y describir las características de la cultura organizacional de la Facultad de Administración de Empresas de la U.N.C.P.para proponer un algoritmo para el cambio cultural.

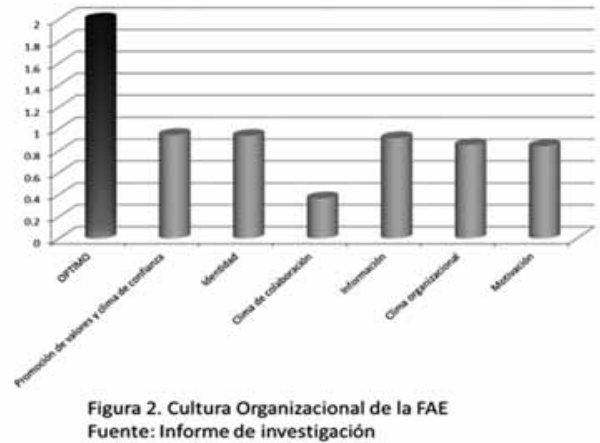

\section{MATERIAL Y METODOS}

La ejecución del trabajo tuvo lugar en la Facultad de Administración de Empresas de la UNCP, utilizando equipos de procesamiento de datos con programas como: Word para y SPSS20 para el procesamiento de los datos que fueron obtenidos mediante una escala de actitud aplicada a una población de 29 miembros de la comunidad FAE ( 26 docentes y 3 personal administrativo). Se utilizó los métodos de inducción y deducción, el análisis y síntesis, aplicados a un trabajo no experimental para el tratamiento de la variable descriptiva cultura organizacional. Las técnicas de la encuesta, la estadística descriptiva y el estadístico $t$, con un nivel de significancia de 0.05 y grado de libertad de 29 ,permitieron rechazar la hipótesis nula y aceptar la hipótesis de investigación.

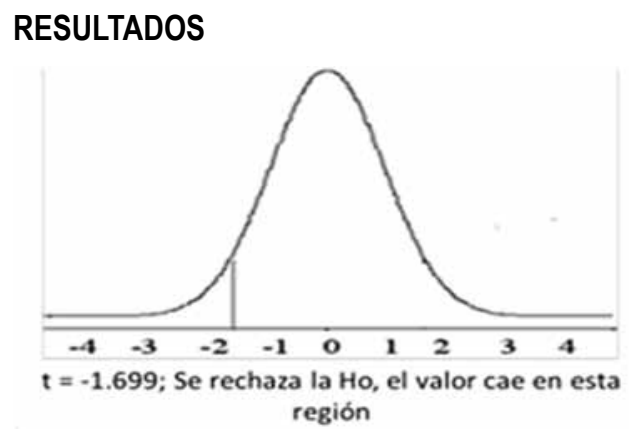

Figura 3. Zonas de aceptación y rechazo de la hipotesis

Los resultados de la investigación con puntaje 
obtenido de 31.90 , permite afirmar que la cultura organizacional de la Facultad de Administración de Empresas es débil, siendo la variable clima de colaboración el más crítico. Es decir que la cultura organizacional no se encuentra definida, y en muchos de sus problemas tiene como causa raíz la cultura y que probablemente existan conflictos internos que no permite encaminarse en los fines de la Facultad.

El estadístico t, con un nivel de significancia de 0.05 y grado de libertad de 29, el estudio concluye que; se rechaza la hipótesis nula y se acepta la hipótesis de investigación, evidenciando que "La cultura organizacional de la Facultad de Administración de Empresas de la Universidad Nacional del Centro del Perú, se singulariza por ser débil; caracterizado por la deficiente promoción de valores y clima de confianza, poca Identidad, deficiente flujo de la información, clima organizacional desfavorable, carencia de motivación».

\section{DISCUSIÓN}

Tal como manifiesta Siliceo y otros, (1999) "el sistema de significados que comparten los miembros y que la distingue de otras organizaciones"; representando las impresiones que tienen los empleados de la compañía, siendo la cultura la que repercute en el comportamiento, productividad y expectativas de los trabajadores; si la Facultad no mejora su clima detectado como débil, tendrá dificultades en alcanzar sus objetivos, especialmente los de mejora continua de la calidad académica, siendo una referencia para los docentes y personal administrativos de los criterios de desempeño (Marquadt, 2002, citado por Ivancevich, 2005).

\section{CONCLUSIONES}

- Con un nivel de significancia de 0.05 y grado de libertad de 29 , se rechaza la hipótesis nula y se acepta la hipótesis de investigación; "La cultura organizacional de la Facultad de Administración de Empresas de la U.N.C.P., se singulariza por ser débil; caracterizado por la deficiente promoción de valores y clima de confianza, poca identidad, deficiente flujo de la información, clima organizacional desfavorable, carencia de motivación». El puntaje acumulado para la Facultad en las seis dimensiones es de 31.90 , lo que permite afirmar que la cultura organizacional de la Facultad de Administración de Empresas es débil, es decir que no se encuentra definida, que muchos de sus problemas tiene como causa raíz la cultura y que probablemente existan conflictos internos que no permite encaminarse en los fines de la Facultad.

- Por la evidencia empírica recogida y evaluada a través de sus dimensiones, se acepta: la cultura organizacional de la Facultad de Administración de Empresas se caracteriza por la deficiente promoción de valores y clima de confianza, poca identidad, pésimo clima de colaboración, existencia de una deficiente comunicación de la información, clima organizacional desfavorable por existencia de grupos que comparten sus propios valores, deficiente motivación.

\section{REFERENCIAS BIBLIOGRÁFICAS}

1. Gibson, James; Ivancevich, Johns y Donnely, James. Las organizaciones: comportamiento, estructura procesos. Editorial: Mc Graw Hill. Octava edición. México, 2001.

2. Ivancevich, J. Administración de recursos humanos. Novena edición. México. Editorial: Mc. Graw Hill, 2005.

3. Davis. Diagnóstico de Cultura Organizacional. medición del clima organizacional de la Escuela Juan José Latorre de la Ciudad de Valparaíso, 1993.

4. Siliceo, Alfonso; Casares, David. y González, José. Liderazgo, valores y cultura organizacional. Editorial: Mc. Graw Hill. México, 1999. 